\title{
Misleading FT4 measurement: Assay-dependent antibody interference
}

\author{
Ingrid Revet ${ }^{1,2}$, Lianne SM Boesten², Jan Linthorst ${ }^{3}$, Elif Yildiz ${ }^{4}$, Johannes W Janssen'1, Yolanda B de Rijke ${ }^{5}$, Arjan Albersen¹,2,6 \\ 'Department of Clinical Chemistry and Hematology, Franciscus Gasthuis, Rotterdam, The Netherlands \\ ${ }^{2}$ General Clinical Laboratory, IJsselland Hospital, Capelle a/d IJssel, The Netherlands \\ ${ }^{3}$ General practitioner's office Linthorst, Berkel en Rodenrijs, The Netherlands \\ ${ }^{4}$ General practitioner's office J.A. de Schepper and E. Yildiz, Rotterdam, The Netherlands \\ ${ }^{5}$ Departments of Clinical Chemistry and Internal Medicine, Erasmus MC University Medical Center, Rotterdam, The Netherlands \\ ${ }^{6}$ Department of Clinical Chemistry and Laboratory Medicine, Leiden University Medical Center, Leiden, The Netherlands \\ *Corresponding author: a.albersen@lumc.nl
}

\begin{abstract}
Introduction: Commonly used free thyroxine (FT4) immunoassays can be falsely elevated due to interference causing misinterpreted thyroid function. We present two cases with high FT4 concentrations due to antibody interference. This study's aim was to investigate the source of the FT4 immunoassay interference and possibility of its removal by two different techniques in order to correct the discrepancy between obtained FT4 values and the patient's clinical status.
\end{abstract}

Materials and methods: Two patients presented at their general practitioners' with elevated FT4 concentrations in combination with a normal and increased thyroid stimulating hormone (TSH) concentrations. Clinical symptoms differed between patients but did not correspond with the hyperthyroid status suggested by the laboratory results. FT4 concentrations from both patients were measured on four common commercial immunoassays and the dialysis method before and after treatment with heterophilic blocking tubes and protein $\mathrm{A} / \mathrm{G}$.

Results: Removal of interfering antibodies using protein A/G resulted in normal FT4 concentrations.

Conclusion: This report illustrates falsely elevated FT4 concentrations due to assay interference on the Immulite immunoassay analyser caused by heterophilic antibodies, which were eliminated by protein $\mathrm{A} / \mathrm{G}$ treatment. We point out the importance of a close collaboration between doctors and the laboratory to avoid unnecessary clinical intervention.

Key words: FT4; thyroid function test; diagnostic errors; interference; immunoassay

\section{Introduction}

In most patients euthyroidism, thyrotoxicosis or hypothyroidism is confirmed with the combination of free thyroxine (FT4) and thyroid stimulating hormone (TSH) measurements. Predominantly, thyroid function tests (TFT) are easily interpreted and correspond with the patient's clinical status. However, in a limited number of cases the TFT and the clinical impression are discordant. A number of factors, including concurrent (non-thyroidal) illness, medication (e.g. amiodarone, thyroxine) and immunoassay interference, can cause these discrepancies (1-3). These factors should be excluded in order to prevent unnecessary diagnostic and therapeutic interventions (4).

Currently, the competitive immunoassay is a common method to determine FT4 concentrations in diagnostic laboratories. These assays measure the unbound thyroxine fraction whereby the vast excess is protein-bound (> 99.5\%). Immunoassay interference can cause false (positive or negative) FT4 results that can lead to misdiagnosis and potentially harmful therapy (5).

Here we describe two patients with a discrepancy between the TFT and the patient's clinical status. 
The aim of the study was to investigate the source and possible removal of the FT4 immunoassay interference in these two patients.

\section{Material and methods}

\section{Case reports}

\section{Patient one}

A 58-year-old woman presented with fatigue and weight gain at the general practitioner (GP). 14 years earlier, she was diagnosed with Hashimoto's autoimmune thyroiditis and has been using levothyroxine since. She was evaluated and thyroid function test revealed a markedly elevated FT4 (28 $\mathrm{pmol} / \mathrm{L}$; reference range $11-21 \mathrm{pmol} / \mathrm{L})$ with normal TSH (1.5 mU/L; reference range 0.16-4.6 mU/L). Two months later, upon return to the GP, levothyroxine dosage was decreased since she complained about palpations in combination with a still markedly increased FT4 (31 pmol/L) and normal TSH (1.1 mU/L). The GP clinically questioned her hyperthyroid status since she also complained about fatigue and weight gain, not in line with hyperthyroidism. Despite lowering her levothyroxine dosage, FT4 measurements kept increasing further now in combination with an increased TSH of 5.8 $\mathrm{mU} / \mathrm{L}$, after which she was referred to a medical specialist who in collaboration with the clinical chemist suspected an interference on the FT4 assay as the culprit (Table 1).

\section{Patient two}

A 65-year-old male presented with abdominal pain, no change in defecation pattern or weight at the GP. He was using levothyroxine for 10 years af- ter being diagnosed with hypothyroidism. Upon evaluation, his thyroid function tests revealed an increased FT4 (36 pmol/L) and TSH ( $7.9 \mathrm{mU} / \mathrm{L})$. The GP contacted the endocrinologist and discussed the clinical symptoms in combination with the laboratory results. They concluded that either there was a problem with the patient's medication compliance or a laboratory discrepancy with the TFT. Upon return to the GP two months later patient's medication compliance was questioned but found adequate and laboratory was repeated with an increased FT4 (29 pmol/L) and TSH (11.5 mU/L). The GP contacted the clinical chemist since the clinical status of the patient did not match the TFT and interference on the FT4 assay was discovered by repeating the analysis on a different platform (Table 1).

\section{Interference testing}

Sera from the patients described were used in this study. Blood was collected in $10 \mathrm{~mL}$ serum vacuum tubes with silica clot activator and polymer gel separator: BD SST II Advance Tube (Becton, Dickinson and Company, Franklin Lakes, NJ, USA). Sera were obtained with informed consent from the patients following national and institutional guidelines.

In addition, commercial sera from two patients with a human anti-mouse antibodies (HAMA) concentration of $2464 \mathrm{ng} / \mathrm{mL}$ and a rheumatoid factor (RF) concentration of $440 \mathrm{lU} / \mathrm{mL}$ were included as known interfering factors for immunoassays (product number 3PH490, lot number SD3158-56 and product number 3PH330, lot number SD314231, respectively, from Scantibodies Laboratory, Santee, USA) (4).

TABLE 1. Initial levels of TSH and FT4 obtained in patients by respectively Immulite, Cobas and DXi platforms

\begin{tabular}{cccccccc}
\hline Patient & Assay & Immulite & $\begin{array}{c}\text { Immulite } \\
\text { reference range }\end{array}$ & Cobas & $\begin{array}{c}\text { Cobas } \\
\text { reference range }\end{array}$ & $\begin{array}{c}\text { DXi } \\
\text { reference range }\end{array}$ \\
\hline \multirow{2}{*}{ Patient 1 } & $\mathrm{FT4}, \mathrm{pmol} / \mathrm{L}$ & 42 & $11-21$ & - & - & 14 & $9-25$ \\
& $\mathrm{TSH}, \mathrm{mU} / \mathrm{L}$ & 0.76 & $0.16-4.60$ & - & - & 0.69 & $0.16-4.60$ \\
\multirow{2}{*}{ Patient 2 } & $\mathrm{FT4}, \mathrm{pmol} / \mathrm{L}$ & 26 & $11-21$ & 15 & $10-21$ & 11 & $9-25$ \\
& $\mathrm{TSH}, \mathrm{mU} / \mathrm{L}$ & 6.40 & $0.16-4.60$ & 7 & $0.40-4.00$ & 6.4 & $0.16-4.60$ \\
\hline
\end{tabular}

FT4 - free thyroxine. TSH - thyroid stimulating hormone. 
FT4 measurements were performed on four different immunoassay analyzers: Cobas Modular E (Roche Diagnostics, Basel, Switzerland), Immulite 2500 (Siemens, Munich, Germany), DXi (Beckman Coulter, Krefeld, Germany) and Vitros ECi (Ortho Clinical Diagnostics, Johnson \& Johnson, Beerse, Belgium) and compared to the equilibrium dialysis as described by Docter et al. (7). The reference ranges for FT4 were as follows: for Cobas Modular 12-22 pmol/L, for Immulite 2500 12-23 pmol/L, for DXi 8-14 pmol/L and for Vitros 11-25 pmol/L. The FT4 reference range for the Dialysis method was determined in-house (14-29 pmol/L). FT4 measurements were normalized by dividing the results with the upper limit of normal (ULN) for each assay (FT4/ULN). Figure 1 schematically presents FT4

A
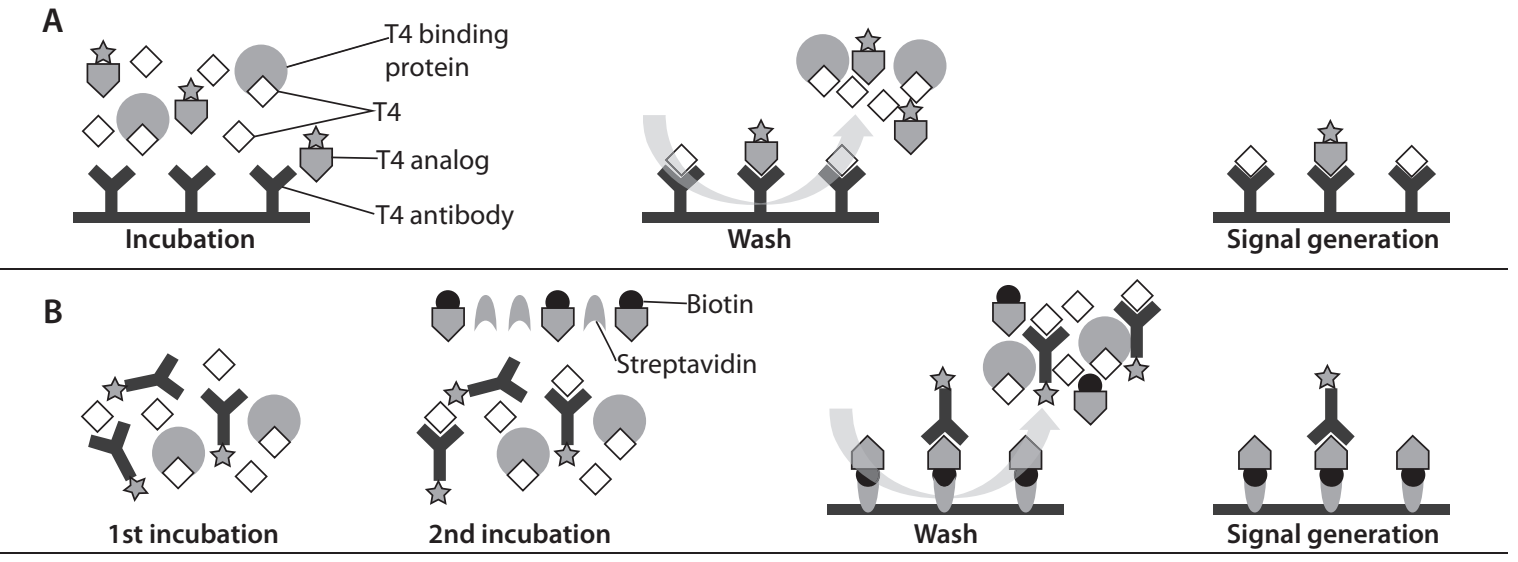

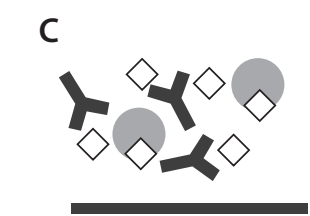

1st incubation

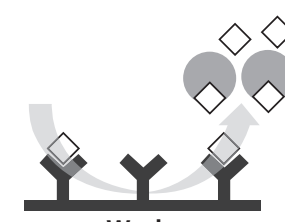

Wash

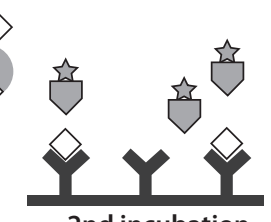

2nd incubation

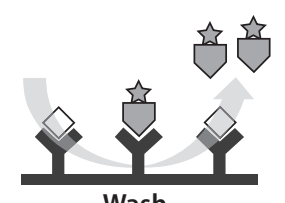

Wash

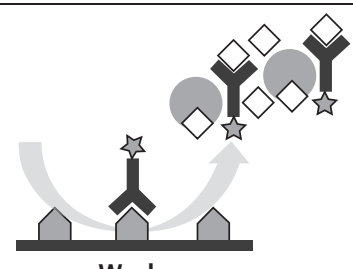

Wash

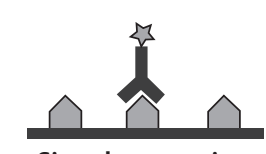

Signal generation

FigURE 1. Schematic presentation of the FT4 measurement commonly used in routine clinical chemistry laboratories.

(A) one-step labelled T4 analog (Immulite, Siemens) in which the patients serum is simultaneously incubated with the labelled T4 analog and competes for binding with the immobilized anti-T4 antibody. After incubation, the unbound fraction is removed by washing and the signal recorded.

(B) The one-step analog two-step incubation (Cobas, Roche Diagnostics) uses a labelled T4 antibody in the first incubation round, followed by a second incubation step in which the T4 analog is added. Only the T4 analog, with or without the labelled T4 antibody, will subsequently bind via biotin and streptavidin and become immobilized. The unbound fraction is removed by washing and the signal is recorded.

(C) The two-step labelled T4 analog method (DXi, Beckman Coulter) introduces the T4 antibody in the first incubation round to the patient's serum. During this incubation step, the T4 antibody becomes immobilized to the solid phase followed by a wash step. In the second incubation step, the labelled T4 analog is allowed to bind to the vacant anti-T4 antibody binding sites, the unbound labelled T4 analog is washed away and the signal recorded.

(D) The one-step labelled antibody method (Vitros, Ortho Clinical Diagnostics) incubates the patient's serum with immobilized T4 analog and uses a labelled anti-T4 antibody. The FT4 in the serum competes for binding with the T4 analog followed by a wash step and signal generation.

In all these designs, the signal generated is inversely proportional to the FT4 concentration. 
measurements on each immunoassay analyzer commonly used in routine clinical chemistry laboratories. FT4 measurements on Cobas Modular E, Immulite 2500, and DXi were performed in duplicate, whereas FT4 measurements on Vitros ECi and equilibrium dialysis was done in singlicate. For the duplicate FT4 measurements, intra-assay variation was determined by taking the $95 \%$ confidence interval of the standard error based on the difference between these measurements.

Treatment of serum with heterophilic blocking tubes (HBT) (Scantibodies Laboratory, Santee, USA) was performed according to manufacturer's instructions. The recombinant fusion protein that combines immunoglobulin (lg) $\mathrm{G}$ binding domains of both Protein A and Protein $G$ (protein A/G) agarose beads; Pierce Protein A/G Plus Agarose (Thermo Scientific, Rockford, USA) were washed and incubated at $4{ }^{\circ} \mathrm{C}$ overnight with sera. The effect of HBT and protein $A / G$ treatment on $\lg G$ and $\lg M$ concentrations of the sera was measured using Immulite 2500 . Antibodies against thyroglobulin (TG) were determined by a sandwich immunoassay (ImmunoCap 250, Phadia Thermoscientific, Uppsala, Sweden). Presence of thyroid hormone autoantibodies (THAA) were tested with agar gel electrophoresis (8).

\section{Results}

The initial patient FT4 and TSH values were obtained using the Immulite analyzer; subsequently, FT4 and TSH were measured on DXi or DXi and Cobas for patient 1 and 2, respectively. Based on the obtained FT4 concentrations, as presented in Table 1, assay interference was suspected. As interference due to antibody presence was suspected and antibody removal was performed.

\section{Antibody removal}

To establish a baseline before removal of the antibodies, we measured IgM and IgG in the patient sera in duplicate. IgM (patient $1=1.19 \mathrm{~g} / \mathrm{L}$ and patient $2=0.95 \mathrm{~g} / \mathrm{L}$ ) and $\mathrm{lgG}$ (patient $1=12.8 \mathrm{~g} / \mathrm{L}$ and patient $2=9.9 \mathrm{~g} / \mathrm{L}$ ) were within the reference range of $0.45-2.30 \mathrm{~g} / \mathrm{L}$ for $\operatorname{lgM}$ and $7.0-15.5 \mathrm{~g} / \mathrm{L}$ for

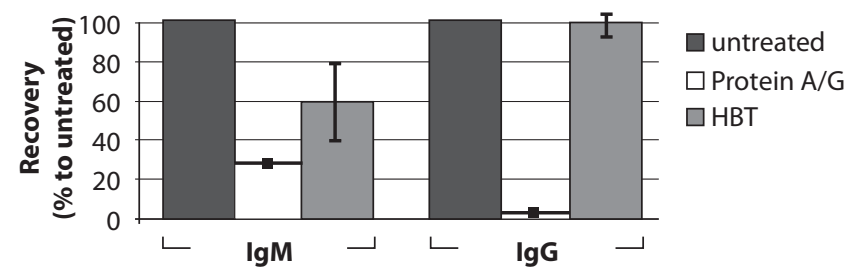

Figure 2. Measurement of IgM and IgG in patients' sera and commercial serum with HAMA or RF. Results obtained by untreated sera are shown with black bars, sera treated by HBT with grey bars and sera treated by protein A/G with white bars. Error bars represent standard deviation of the mean.

IgG. After either Protein A/G or HBT treatment, IgM and IgG were measured again in duplicate to investigate the effectiveness of antibody removal. Analysis of all sera showed a $40 \%$ decrease of IgM concentrations upon HBT treatment whereas no significant effect on the $\lg G$ concentrations was detected (Figure 2). Only a quarter of the original IgM concentrations could be detected after protein $A / G$ treatment whereas almost no lgG concentrations were detected (Figure 2).

Subsequent analysis of the patient sera showed elevated FT4 with the Immulite assay in comparison with the DXi, Cobas, Vitros and Dialysis method for patient 1 and 2 (Figure $3 A$ and $B$ ). No interference of HAMA (2464 $\mathrm{ng} / \mathrm{mL})$ or RF $(440 \mathrm{IU} / \mathrm{mL})$ was detected in the FT4 assay performed on 4 different analyzers or using the dialysis method (Figure $3 \mathrm{C}$ and D). Removal of antibodies with protein $A / G$ treatment resulted in lower FT4 concentrations for the Immulite and Cobas assays for sera 1 and 2, whereas no effect or a slight increase was seen in Dxl, Vitros and Dialysis methods for these sera. FT4 results after treatment with $\mathrm{HBT}$ gave similar results as untreated sera (Figure 3 ).

$\mathrm{TSH}$, total triiodothyronine and total thyroxine showed no aberrant results on the 3 different analyzers, indicating that interfering antibodies do not necessarily give aberrant results on the whole thyroid panel used in current hospital laboratories.

In order to determine the source of the interfering antibody, anti-thyreoglobuline (anti-TG) and THAA were measured in sera 1 and 2 . Both samples contained high concentrations of anti-TG $(388 \mathrm{U} / \mathrm{mL}$ 
A

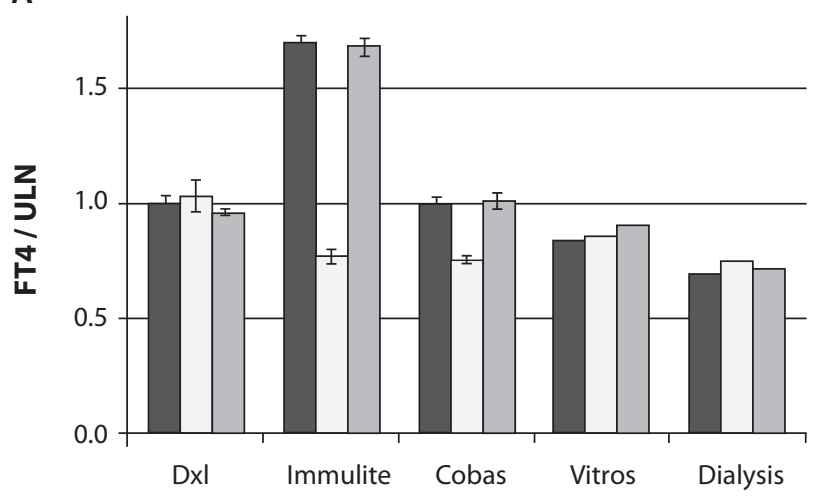

C

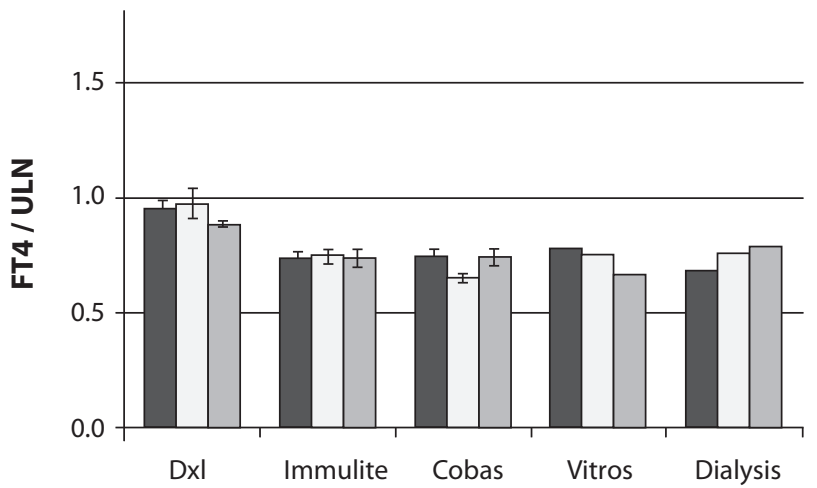

B

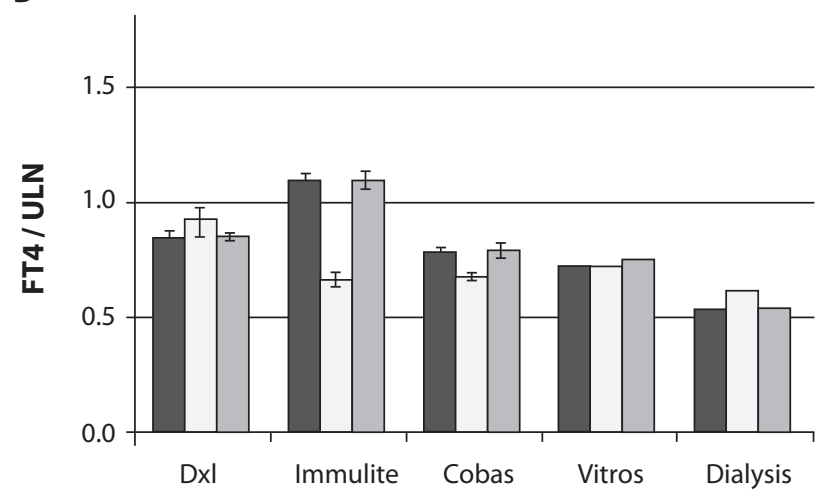

D

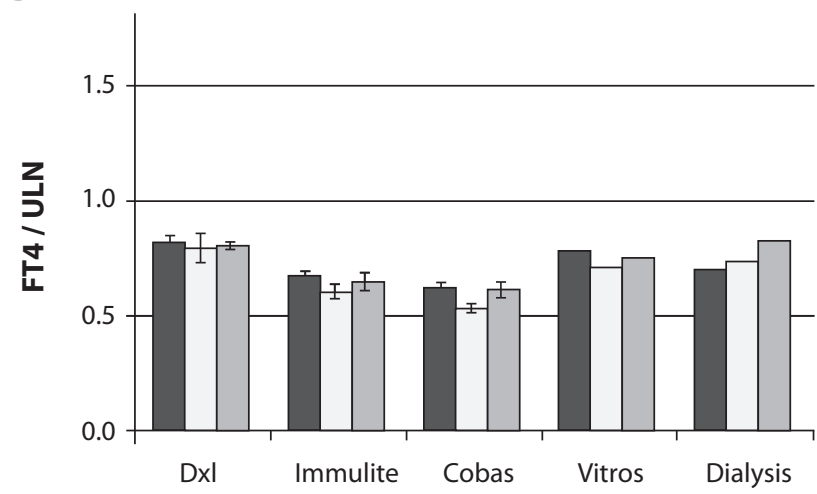

FigURE 3. Measurements of FT4 by four different analyzers and the dialysis method of patient 1 (A), patient 2 (B), commercial serum with HAMA (C) and commercial serum with RF (D). Results obtained by untreated sera are shown with black bars, sera treated by HBT with grey bars and sera treated by protein $A / G$ with white bars. Error bars represent intra-assay variation.

and $66 \mathrm{U} / \mathrm{mL}$ for patient 1 and 2, respectively, with a reference value $<40 \mathrm{U} / \mathrm{mL}$ ) but did not contain THAA. Therefore we speculated anti-TG might interfere with the Immulite assay in particular. However a random selection of 26 sera with high titers of anti-TG showed no discordance between Immulite and Roche FT4 results (data not shown).

\section{Clinical follow-up}

After detection of FT4 assay interference in the first patient, levothyroxine therapy was increased to her regular dosage. Upon re-evaluation 3 months later she presented with normal TSH (0.81 $\mathrm{mU} / \mathrm{L})$, normal FT4 (13 pmol/L) and clinically euthyroid. In the second patient the interference was discovered in an earlier stage. Upon re-evaluation he presented with normal TSH $(2.5 \mathrm{mU} / \mathrm{L})$ and clinically euthyroid. For patients FT4 concentrations were no longer monitored using the Immulite method because of the interference.

\section{Discussion}

In this report we performed a side-by-side comparison of the efficacy of two interfering antibody removal techniques in FT4 assays across multiple immunoassay analyzers. We show that protein $A / G$ treatment prior to analysis performed superiorly in comparison with the commonly used HBT treatment to remove interfering antibodies. Our data demonstrates its effectiveness for the first time by $\lg \mathrm{M}$ and IgG measurement before and after protein $A / G$ treatment.

This study showed that in the Immulite immunoassay discrepant FT4 results in two patients were due to interfering antibodies. Several interferenc- 
es of the FT4 assay have been described including heterophilic antibodies (4), human anti mouse antibodies (HAMA) (4), thyroid hormone auto-antibodies (THAA), albumin variants (familial dysalbuminaemic hyperthyroxinaemia) and heparin use (9-14). Since patients did not use heparin it was postulated that an antibody caused the grossly elevated FT4 results on the Immulite 2500 in comparison with other analyzers and equilibrium dialysis. Several different immunoassay tests have been reported to give aberrant results when interfering antibodies are present (15-18). Antibody interference was eliminated by treatment with protein A/G sepharose but not with HBT. Furthermore, we have demonstrated that protein $A / G$ treatment has little effect on FT4 immunoassay measurement.

When FT4 immunoassay interference is suspected, retesting with a different commercial assay is a useful strategy $(18,19)$. However, comparison of FT4 results between different methods can be challenging $(20,21)$. One should be aware of these differences between laboratory methods and use the appropriate reference intervals when interpreting results.

Different strategies have been reported for removal of interfering antibodies $(1,4,10,22)$. Upon treatment of the sera we were able to partially remove IgM antibodies with HBT treatment whereas Protein $A / G$ removes both IgM partially and IgG completely. The latter treatment led to normal FT4 concentrations on the Immulite assay comparable to concentrations found using DXi, Cobas, Vitros and dialysis methods. We chose to treat with protein $A / G$ instead of polyethylene glycol precipitation (PEG) because of the specific ability of protein $A / G$ to bind and remove only immunoglobulins. Known disadvantages for PEG treatment are the non-specific nature causing precipitation of proteins and ability to interfere with immunoassays (23). Since the protein A/G treatment corrected the FT4 results on the Immulite assay, we speculated THAA might be the culprit. THAA have been reported to be of the IgG class, which is almost completely removed by protein A/G treatment (24). However no THAA were detected in our sera. Since THAA coexists with antibodies to thyroglobulin, we screened an additional 26 sera on Immulite and Cobas but no discrepancies were found. We speculate that an antibody interferes with the Immulite assay since partial lgM and near complete IgG antibodies removal corrected the high results on the Immulite assay. Since none of the other assays are influenced by this antibody we postulate two possible hypotheses why the Immulite assay (one-step method) produced aberrant high FT4 results. Firstly, the interfering antibodies interacts with the capture antibodies and somehow prevents tracer, but not FT4 binding. Antibodies in the remaining assays differ and therefore do not interact with the interfering substance. The exact binding properties of the antibodies used are proprietary information and therefore could not be compared to possibly identify the source of interference. Secondly, the interfering antibody binds the tracer directly and prevents the tracer from binding to the capture antibody. Therefore, interfering antibody-tracer complex is washed away resulting in falsely elevated FT4 result (competitive immunoassay). This is only possible in a one-step assay since the interfering substance remains present during the entire procedure. The other one-step immunoassay, Cobas Modular E, has two consecutive incubations. Firstly, serum and capture antibody are incubated followed by a second incubation whereby tracer is added. The test is completed by a wash step. This assay is possibly also affected by the interfering antibody whereby protein A/G treatment reduces FT4 concentrations. The DXi method is a two-step assay with a separate washing step that potentially removes the interfering substance before analysis (Figure 1) and consequently avoiding direct contact with the tracer. The Vitros method uses a one-step principle like the Immulite assay but interference is seen only with the Immulite. Our hypothesis is that the antibody that interferes in the Immulite and Cobas assays is somehow blocked by the blocking agents used in the Vitros assay or the labeled anti-T4 antibody is modified in such a way that it is less likely to cause aberrant results by antibodies present in human serum. Since the dialysis method is independent of binding proteins and no antibodies are used the interfering immunoglobulin will not alter the results of the dialysis FT4 measurement. 
HBT treatment might be an effective procedure in routine laboratories in case immunoassays results are suspected to be influenced by interfering antibodies $(25,26)$. However, most commercial immunoassays already use blocking agents. This possibly explains the fact no differences were observed in HBT treated and untreated HAMA serum (Figure 2C).

Looking for the source of the interference we included sera that contained HAMA and RF antibodies. These have been reported to interfere with hormone assays (1) but in our study we did not find any effect on the FT4 measurements on the Immulite Modular, DXi, Vitros analyzers or the dialysis method.

In conclusion the interfering antibody in this study is only able to disturb the FT4 results in the Immulite assay. By comparing FT4 result of different commercial immunoassays interference was confirmed. HBT were not able to remove the interfer-

\section{References}

1. Bolstad N, Warren DJ, Bjerner J, Kravdal G, Schwettmann $L$, Olsen $K H$, et al. Heterophilic antibody interference in commercial immunoassays; a screening study using paired native and pre-blocked sera. Clin Chem Lab Med 2011;49:2001-6. http://dx.doi.org/10.1515/CCLM.2011.702.

2. Cartwright $D$, O'Shea $P$, Rajanayagam $O$, Agostini $M$, Barker P, Moran C, et al. Familial dysalbuminemic hyperthyroxinemia: a persistent diagnostic challenge. Clin Chem 2009;55:1044-6. http://dx.doi.org/10.1373/ clinchem.2008.120303.

3. Siccama R, Balk AH, de Herder WW, van Domburg R, Vantrimpont $P$, van Gelder T. Amiodarone therapy before heart transplantation as a predictor of thyroid dysfunction after transplantation. J Heart Lung Transplant 2003;22:857-61. http://dx.doi.org/10.1016/S1053-2498(02)01149-X.

4. Despres N, Grant AM. Antibody interference in thyroid assays: a potential for clinical misinformation. Clin Chem 1998;44:440-54.

5. Caballero A, Corcoy $R$, Negredo E, Rodriguez-Espinosa J. Autoantibodies against thyroid hormones can lead to an erroneous diagnosis and potentially harmful treatment. Ann Clin Biochem 1998;35:152-3. http://dx.doi. org/10.1177/000456329803500125. ing substance judging by the FT4 levels above the reference levels after treatment in the Immulite assay. After protein A/G treatment the FT4 results dropped to levels within reference, comparable to those measured with the other methods. Leading to the conclusion that protein $A / G$ treatment effectively removes the interfering antibody. In addition this study shows the importance of proper collaboration between a doctor and clinical chemist when laboratory results are not in line with the clinical presentation.

\section{Acknowledgements}

We would like to thank Hans van Toor, Johan Tak, Heleen Angenent, Ton Kerdel, Rene Ysselstijn for technical assistance and Erwin Birnie for statistical advice.

\section{Potential conflict of interest}

None declared.
6. Demers LM, Spencer CA. Laboratory medicine practice guidelines: laboratory support for the diagnosis and monitoring of thyroid disease. Clin Endocrinol (Oxf)2003;58:13840. http://dx.doi.org/10.1046/j.1365-2265.2003.01681.x.

7. Docter $R$, van Toor $H$, Krenning EP, de Jong M, Hennemann $G$. Free thyroxine assessed with three assays in sera of patients with nonthyroidal illness and of subjects with $a b$ normal concentrations of thyroxine-binding proteins. Clin Chem 1993;39:1668-74.

8. Hennemann $G$, Docter $R$, Krenning EP, Bos G, Otten M, Visser TJ. Raised total thyroxine and free thyroxine index but normal free thyroxine. A serum abnormality due to inherited increased affinity of iodothyronines for serum binding protein. Lancet 1979;1:639-42. http://dx.doi.org/10.1016/ S0140-6736(79)91080-8.

9. Loh TP, Leong SM, Loke KY, Deepak DS. Spuriously elevated free thyroxine associated with autoantibodies, a result of laboratory methodology: case report and literature review. Endocr Pract 2014;20:e134-9. http://dx.doi.org/10.4158/ EP14059.CR.

10. Zouwail SA, O'Toole AM, Clark PM, Begley JP. Influence of thyroid hormone autoantibodies on 7 thyroid hormone assays. Clin Chem 2008;54:927-8. http://dx.doi.org/10.1373/ clinchem.2007.099770. 
11. Petitpas I, Petersen $C E$, Ha CE, Bhattacharya AA, Zunszain $P A$, Ghuman J, et al. Structural basis of albumin-thyroxine interactions and familial dysalbuminemic hyperthyroxinemia. Proc Natl Acad Sci U S A 2003;100:6440-5. http://dx.doi. org/10.1073/pnas.1137188100.

12. Ross HA, de Rijke YB, Sweep FC. Spuriously high free thyroxine values in familial dysalbuminemic hyperthyroxinemia. Clin Chem 2011;57:524-5. http://dx.doi.org/10.1373/ clinchem.2010.158170.

13. Jaume JC, Mendel CM, Frost PH, Greenspan FS, Laughton CW. Extremely low doses of heparin release lipase activity into the plasma and can thereby cause artifactual elevations in the serum-free thyroxine concentration as measured by equilibrium dialysis. Thyroid 1996;6:79-83. http://dx.doi. org/10.1089/thy.1996.6.79.

14. Stockigt JR. Free thyroid hormone measurement. A critical appraisal. Endocrinol Metab Clin North Am 2001;30:265-89. http://dx.doi.org/10.1016/S0889-8529(05)70187-0.

15. Shimon I, Pariente C, Shlomo-David J, Grossman Z, Sack J. Transient elevation of triiodothyronine caused by triiodothyronine autoantibody associated with acute EpsteinBarr-virus infection. Thyroid 2003;13:211-5. http://dx.doi. org/10.1089/105072503321319530.

16. Verdickt L, Maiter D, Depraetere L, Gruson D. TSH-assay interference: still with us. Clin Lab 2012;58:1305-7.

17. Gessl A, BluemI S, Bieglmayer C, Marculescu R. Anti-ruthenium antibodies mimic macro-TSH in electrochemiluminescent immunoassay. Clin Chem Lab Med 2014;52:1589-94. http://dx.doi.org/10.1515/cclm-2014-0067.

18. Kwok JS, Chan IH, Chan MH. Biotin interference on TSH and free thyroid hormone measurement. Pathology 2012;44:278-80. http://dx.doi.org/10.1097/ PAT.0b013e3283514002.
19. Lewandowski KC, Dabrowska K, Lewinski A. Case report: When measured free T4 and free T3 may be misleading. Interference with free thyroid hormones measurements on Roche(R) and Siemens(R) platforms. Thyroid res 2012;5:11. http://dx.doi.org/10.1186/1756-6614-5-11.

20. Williams F, Hume R. The measurement, definition, aetiology and clinical consequences of neonatal transient hypothyroxinaemia. Ann Clin Biochem 2011;48:7-22. http:// dx.doi.org/10.1258/acb.2010.010174.

21. Springer D, Bartos $V$, Zima T. Reference intervals for thyroid markers in early pregnancy determined by 7 different analytical systems. Scand J Clin Lab Invest 2014;74:95-101. http://dx.doi.org/10.3109/00365513.2013.860617.

22. Martins TB, Jaskowski TD, Mouritsen CL, Hill HR. An evaluation of the effectiveness of three immunoglobulin $G$ ( $\mathrm{IgG}$ removal procedures for routine IgM serological testing. Clin Diagn Lab Immunol 1995;2:98-103.

23. Fahie-Wilson M, Halsall D. Polyethylene glycol precipitation: proceed with care. Ann Clin Biochem 2008;45:233-5. http:// dx.doi.org/10.1258/acb.2008.007262.

24. Benvenga S, Bartolone L, Squadrito S, Trimarchi F. Thyroid hormone autoantibodies elicited by diagnostic fine needle biopsy. J Clin Endocrinol Metab 1997;82:4217-23. http:// dx.doi.org/10.1210/jc.82.12.4217.

25. Papapetrou PD, Polymeris A, Karga H, Vaiopoulos G. Heterophilic antibodies causing falsely high serum calcitonin values. J Endocrinol Invest 2006;29:919-23. http://dx.doi. org/10.1007/BF03349197.

26. Cappy H, Pigny P, Leroy-Billiard M, Dewailly D, Catteau-Jonard S. Falsely elevated serum antimullerian hormone level in a context of heterophilic interference. Fertil Steril 2013;99:1729-32. http://dx.doi.org/10.1016/j.fertnstert.2012.12.037. 